Moroccan J. of Pure and Appl. Anal. (MJPAA)

Volume 5(1), 2019, Pages 63-85

ISSN: Online 2351-8227 - Print 2605-6364

DOI 10.2478/mjpaa-2019-0006

\title{
On a Generalized Raised Cosine Distribution: Some Properties, Characterizations and Applications
}

\author{
M. Ahsanullah ${ }^{1, a}$, M. ShaKiL ${ }^{2, b}$ and B.M. Golam Kibria ${ }^{3, c}$
}

ABstract. In this paper, we introduced a generalization of the raised cosine distribution. We also provided its several distributional properties and characterizations, including percentiles and some applications.

2010 Mathematics Subject Classification.60E05, 62E10, 62E15, 62G30.

Key words and phrases. Characterizations; Generalized raised cosine distribution; Order Statistics; Truncated Moment.

\section{Introduction}

Several symmetric continuous probability distributions involving trigonometric functions have been developed by many researchers among them the raised cosine distribution (RCD) is noteworthy, because of its wide applications in many real world lifetime data. See, for examples, King [11], Kyurkchiev and Kyurkchiev [13], Rinne [16] and Willink [18], and among others. Ahsanullah and Shakil [7] studied the characterizations of the raised cosine distribution by truncated moment. The generalizations of continuous probability distributions have received a lot of attention in recent years, where the number of parameters is increased so that these distributions become more flexible and can be applied to wide number of real world data. In this paper, we introduce a generalization of the raised cosine distribution (which we denote as GENRCD), by adding another location and scale parameters. It is observed that our proposed new distribution bears most of the properties of symmetric distributions in reliability and life testing context. The organization of the paper is as follows. In Section 2, the generalization of the raised cosine distribution is introduced, along with the graphs of its probability density and cumulative

Received August 04, 2019 - Accepted September 12, 2019.

(C) The Author(s) 2017. This article is published with open access by Sidi Mohamed Ben Abdallah University.

${ }^{1}$ Rider University, Lawrenceville, NJ, USA

${ }^{2}$ Miami Dade College, Hialeah, FL, USA

${ }^{3}$ Florida International University, Miami, FL, USA

a e-mail:ahsan@rider.edu

${ }^{b}$ e-mail: mshakil@mdc.edu

c e-mail: kibriag@fiu.edu . 
distribution functions. In Section 3, several basic distributional properties of GENRCD are provided, including some reliability properties. Section 4 contains characterization results. We have computed the percentiles of the new distribution in Section 5. In Section 6, we give estimation of parameters and have shown some applications in Section 7. Concluding remarks are given in Section 8.

\section{A Generalized Raised Cosine Distribution (GENRCD)}

We propose the following generalized raised cosine distribution, that is, a continuous random variable, $X$ is said to have a generalized raised cosine distribution, $X \sim \operatorname{GENRCD}(\mu, \sigma)$, if its probability density function (pdf) is given by

$$
f(x)=C\left(\alpha+\lambda \cos \left(\frac{(x-\mu) \pi}{\sigma}\right)\right),
$$

where $\sigma>0,0<|\lambda| \leq|\alpha|<\infty$ and $-\infty<\mu<\infty$ are real numbers, with $\mu-\sigma \leq x \leq \mu+\sigma, \alpha$ denotes the location parameter, $\lambda$ denotes the scale parameter, and $C$ is the normalizing constant. It can easily be seen that, after integrating (2.1) with respect to $x$ from $\mu-\sigma$ to $\mu+\sigma$, the normalizing constant $C$ is given by $C=\frac{1}{2 \alpha \sigma}$. By proper choice of parameters, it is observed that the pdf (2.1) contains the well-known raised cosine distribution $X \sim R C D(\mu, \sigma)$, the standard raised cosine distribution $X \sim R C D(0,1)$, and the cardioid distribution. The cumulative distribution (cdf) $F(x)$ corresponding to the pdf (2.1) is easily given by

$$
F(x)=\frac{1}{2}\left[1+\frac{x-\mu}{\sigma}+\frac{\lambda}{\alpha \pi} \sin \left(\frac{(x-\mu) \pi}{\sigma}\right)\right] .
$$

To describe the shapes of the generalized raised cosine distribution,

$$
X \sim \operatorname{GENRCD}(\alpha, \lambda, \mu, \sigma),
$$

the graphs of the pdf (2.1) and cdf (2.2) are given in Figure 1 and Figure 2 respectively, for some selected values of the parameters. From these figures, the effects of the parameters can easily be observed. We note that, $X \sim \operatorname{GENRCD}(\alpha, \lambda, \mu, \sigma)$ since, is an even function, the pdf of $X \sim \operatorname{GENRCD}(\alpha, \lambda, \mu, \sigma)$ is symmetric about the mean $\mu=0$. Similarly, we can draw graphs of the pdf of $X \sim \operatorname{GENRCD}(\alpha, \lambda, \mu, \sigma)$ for other values of the parameters. 


\section{Graphs of the pill of the Generalzed Raised Cosine Distibution}

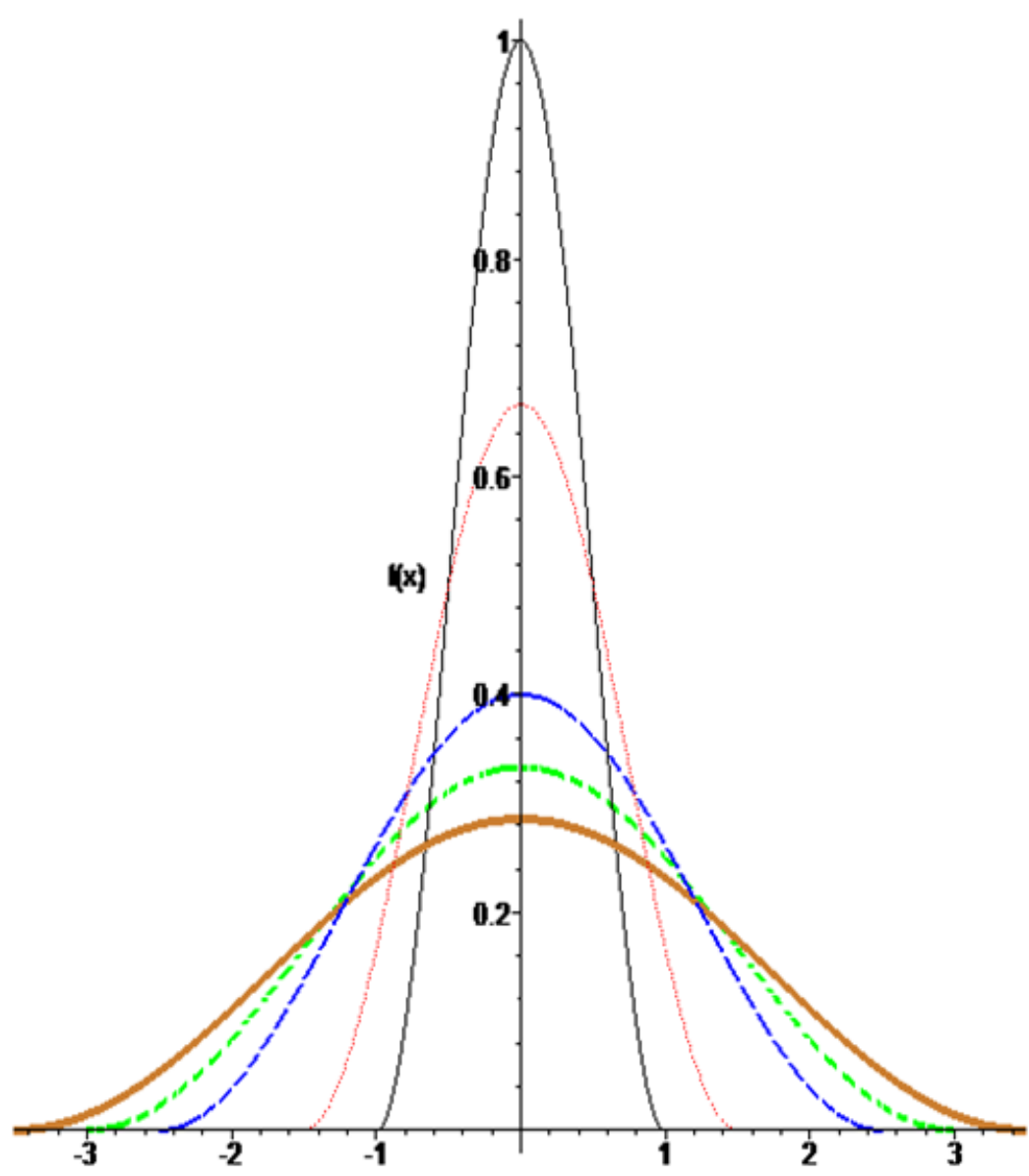

$\mathbf{x}$

singa=1.5, mur 0 , apha $=1$, bambda $=1$

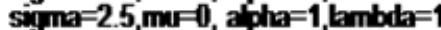
sin ma=3.5, mu=0, a pha $=5$, lambda=1 sinma=1, muld, apha $=1$, lambda=1 sigma =3,mu=0, a'pha=1, lambla=1

Figure 1. PDF $f(x)$ of $X \sim \operatorname{GENRCD}(\alpha, \lambda, \mu, \sigma)$. 


\section{Graphs of the cell of the Generalized Raised Cosine Distribution}

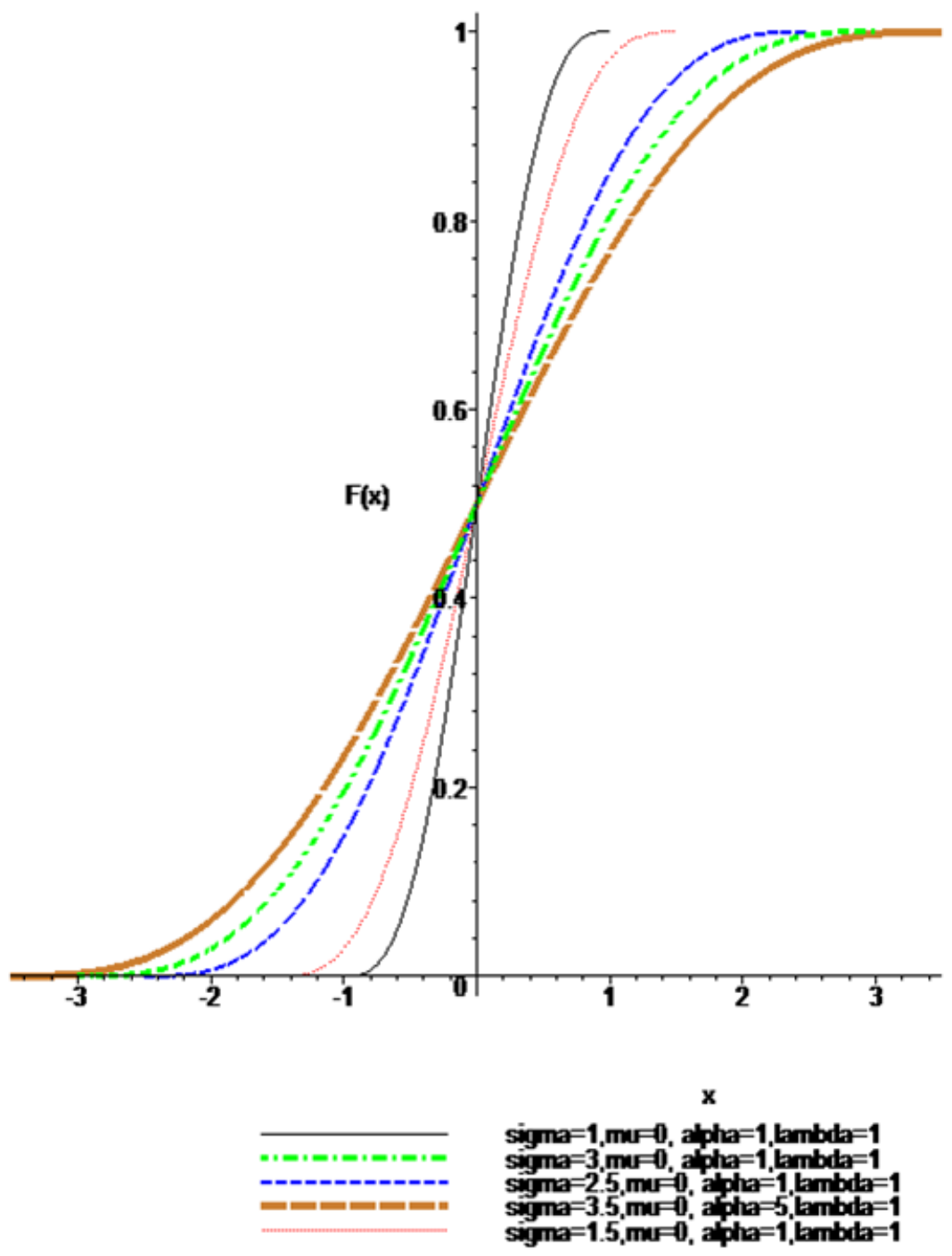

Figure 2. $\operatorname{CDF} F(x)$ of $X \sim G E N R C D(\alpha, \lambda, \mu, \sigma)$.

\section{Distributional Properties}

We present some distributional properties here.

3.1. Mean. It can easily be seen that the mean of $X \sim \operatorname{GENRCD}(\alpha, \lambda, \mu, \sigma)$, is given by

$$
\text { Mean }=E(x)=\int_{\mu-\sigma}^{\mu+\sigma} x\left[\frac{1}{2 \alpha \sigma}\left(\alpha+\lambda \cos \left(\frac{(x-\mu) \pi}{\sigma}\right)\right)\right] d x=\mu \text {. }
$$

3.2. Median. Since the graph of the pdf of $X \sim \operatorname{GENRCD}(\alpha, \lambda, \mu, \sigma)$ is symmetric about the mean $\mu$, its median $\mathrm{MD}$ is also be given by $M D=\mu$. 
3.3. Mode. Further, the mode of the generalized raised cosine distribution is the value of $x=x_{m}$ (say), for which its pdf (2.1) is maximum. Now, differentiating (2.1) with respect to $x$, we have

$$
\frac{d f(x)}{d x}=-\frac{\pi \lambda}{2 \alpha \sigma^{2}} \sin \left(\frac{\pi(x-\mu)}{\sigma}\right) .
$$

Now, $\frac{d f(x)}{d x}=0$ has the unique solution $\mu$. Since $\frac{d f(x)}{d x}>0$ for $x<\mu$ and $<0$ for $x>\mu$, the pdf $f(x)$ increases as $x \rightarrow \mu$ and decreases as $x \rightarrow \mu+\sigma$. Therefore, the generalized raised cosine distribution is unimodal, and mode $=\mu$.

3.4. Inflection Points. By solving the equation

$$
\frac{d^{2} f(x)}{d x^{2}}=-\frac{\pi^{2} \lambda}{2 \alpha \sigma^{3}} \cos \left(\frac{\pi(x-\mu)}{\sigma}\right)
$$

for $x$, it can easily be seen that the pdf (2.1) is concave up in the intervals $\left(\mu-\sigma, \mu-\frac{\sigma}{2}\right) \cup\left(\mu+\frac{\sigma}{2}, \mu+\sigma\right)$ and concave down in the interval, $\left(\mu-\frac{\sigma}{2}, \mu+\frac{\sigma}{2}\right)$ and, hence, its inflection points are given by $x=\mu \pm \frac{\sigma}{2}$.

3.5. Moment Generating Function. The moment generating function (MGF), $M_{x}(t)$, is given by

$$
M_{x}(t)=\int_{\mu-\sigma}^{\mu+\sigma} e^{t x} \frac{1}{2 \alpha \sigma}\left[\left(\alpha+\lambda \cos \left(\frac{(x-\mu) \pi}{\sigma}\right)\right)\right] d x
$$

Substituting $\frac{\pi(x-\mu)}{\sigma}=z$, and using $\int e^{a x} \cos b x d x=\frac{e^{a x}}{a^{2}+b^{2}}(a \cos b x+b \sin b x)+C$, and $\sinh (z)=\frac{1}{2}\left(e^{z}-\right.$ $\left.e^{-z}\right)$, we have

$$
M_{x}(t)= \begin{cases}1 & , t=0 \\ \frac{\left[\alpha \pi^{2}-\sigma^{2} t^{2}(\lambda-\alpha)\right] e^{\mu t} \sinh (\sigma t)}{\alpha \sigma t\left(\pi^{2}+\sigma^{2} t^{2}\right)} & , t \neq 0 .\end{cases}
$$

Remark 3.1. Using the above expression for the MGF, $M_{x}(t)$, and by applying the L'Hospital's rule, we have $E(x)=$ $\lim _{t \rightarrow 0} \frac{d M_{x}(t)}{d t}=\mu$, which is true since we already have $E(x)=\mu$, as shown above.

3.6. Characteristic Function. The characteristic function $(\mathrm{CF}), \Phi_{x}(t)$, is given by

$$
\Phi_{x}(t)=M_{x}(i t)= \begin{cases}1 & , \quad t=0 \\ \frac{\left[\alpha \pi^{2}+\sigma^{2} t^{2}(\lambda-\alpha)\right] e^{i \mu t} \sin (\sigma t)}{\alpha \sigma t\left(\pi^{2}-\sigma^{2} t^{2}\right)}, & t \neq 0, i=\sqrt{-1} .\end{cases}
$$

3.7. Reliability. Recalling the definition of the hazard (or failure) rate for non-repairable populations as the instantaneous rate of failure for the survivors to time, say, $x$, during the next instant of time, the survival (or reliability), the hazard (or failure) rate functions, and cumulative hazard function $H(x)$ of $X \sim \operatorname{GENRCD}(\alpha, \lambda, \mu, \sigma)$ are respectively given by

and

$$
\begin{gathered}
R(x)=1-F(x)=\frac{1}{2}\left[1-\frac{x-\mu}{\sigma}-\frac{\lambda}{\alpha \pi} \sin \left(\frac{(x-\mu) \pi}{\sigma}\right)\right], \\
h(x)=\frac{f(x)}{1-F(x)}=\frac{\alpha+\lambda \cos \left(\frac{(x-\mu) \pi}{\sigma}\right)}{\alpha \sigma\left[1-\frac{x-\mu}{\sigma}-\frac{\lambda}{\alpha \pi} \sin \left(\frac{(x-\mu) \pi}{\sigma}\right)\right]},
\end{gathered}
$$

$$
H(x)=-\ln (R(x))=\ln (2 \alpha \pi)-\ln (\lambda)-\ln \left[\frac{\alpha \pi}{\lambda}\left(1-\frac{x-\mu}{\sigma}\right)-\sin \left(\frac{\pi(x-\mu)}{\sigma}\right)\right] .
$$


Since the hazard rate, $h(x)$, has the following property: $h^{\prime}(x) \geq 0$, that is, $f^{\prime}(x)[1-F(x)]+[f(x)]^{2} \geq 0$, for all $x$ in $\mu-\sigma \leq x \leq \mu+\sigma$, and for all values of the parameters, therefore $X \sim \operatorname{GENRCD}(\alpha, \lambda, \mu, \sigma)$ has an increasing failure rate (IFR). Also, we observe that, since it can easily be seen that $h^{\prime \prime}(x) \geq 0$, for all $x$ in $\mu-\sigma \leq x \leq \mu+\sigma$, and for all values of the parameters, the hazard rate, $h(x)$, is concave up and bathtub shaped. It is also sometimes useful to find the average failure rate function ( AFR ), over any interval, say, $(0, t)$, that averages the failure rate over the interval. Thus, for the proposed distribution, it is given by

$$
\begin{aligned}
\operatorname{AFR}(0, t) & =\operatorname{AFR}(t)=-\frac{1}{t} \ln (R(t)) \\
& =-\frac{1}{t}\left[\ln \left(2 \alpha \pi-\ln (\lambda)-\ln \left[\frac{\alpha \pi}{\lambda}\left(1-\frac{t-\mu}{\sigma}-\sin \left(\frac{\pi(t-\mu)}{\sigma}\right)\right)\right]\right]^{\prime}\right.
\end{aligned}
$$

which in view of the expansion of logarithmic function as a power series, is seen to be positive for all $x$ in $\mu-\sigma \leq x \leq \mu+\sigma$, irrespective of the values of the parameters, and hence $X \sim \operatorname{GENRCD}(\alpha, \lambda, \mu, \sigma)$ is Increasing Failure Rate on Average (IFRA).

The graph of the cumulative hazard function $H(x)$ is given in Figure 3 for some selected values of parameters. The effects of the parameters are obvious from the figure. The increasing and bathtub shape behaviors of $H(x)$ are also evident from the Figure 3. 


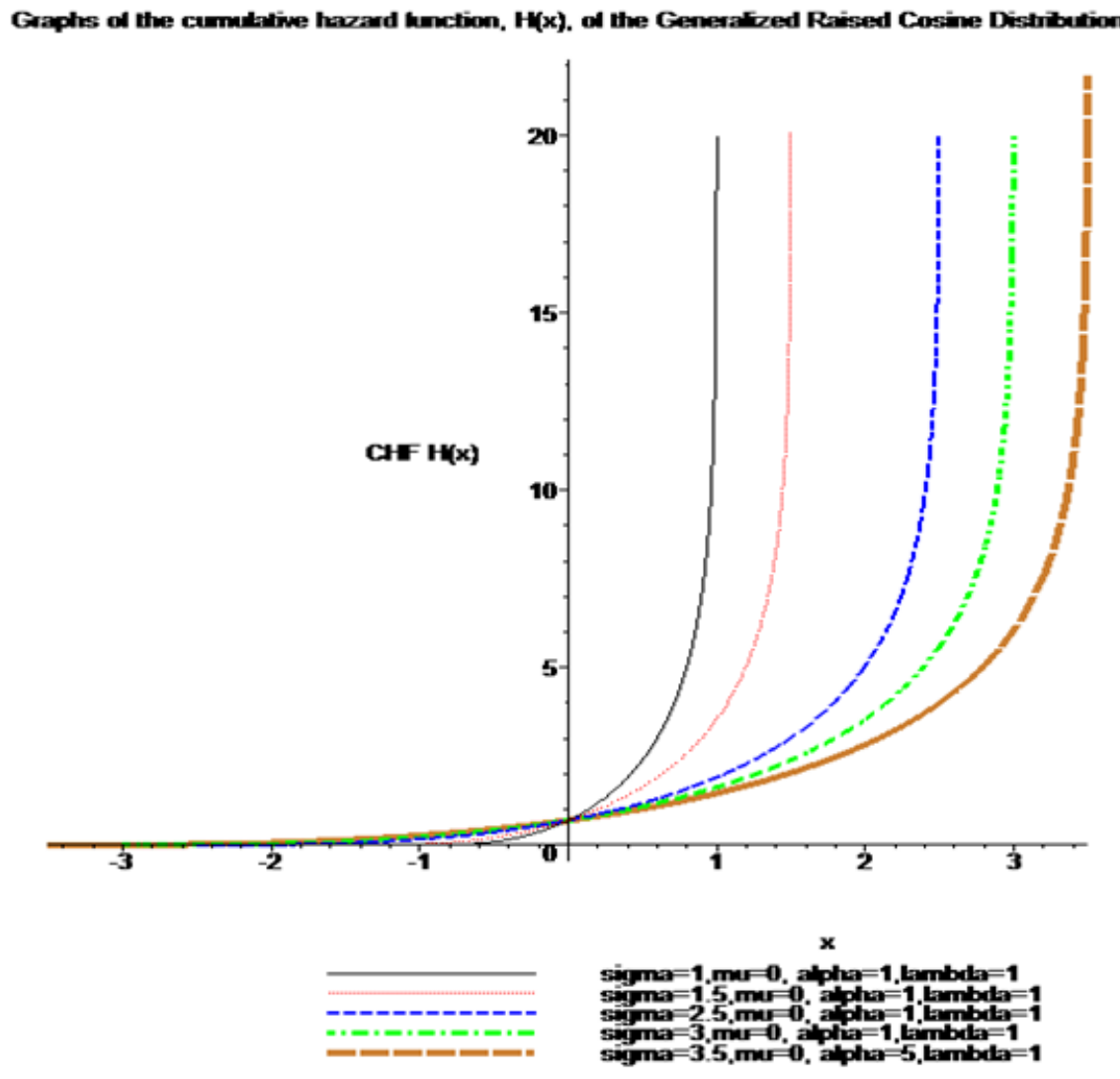

Figure 3. Cumulative Hazard Function, $H(x)$, of $X \sim \operatorname{GENRCD}(\alpha, \lambda, \mu, \sigma)$.

\section{Characterizations}

Several researchers have investigated the characterizations of probability distributions at different times; see, for example, Ahsanullah [2], Ahsanullah et al. [4], Ahsanullah et al. [5, 6], Galambos and Kotz [10], Kotz and Shanbhag [12], and Nagaraja [14], among others. In this section, we present the characterizations of the generalized raised cosine distribution, $X \sim \operatorname{GENRCD}(\alpha, \lambda, \mu, \sigma)$, by truncated first moment, order statistics and upper record values. For this, we will need the following assumption and lemmas.

Assumption 4.1: Suppose the random variable $X$ is absolutely continuous with cumulative distribution function (cdf) $F(x)$ and probability density function (pdf) $f(x)$. We assume that $\gamma=\sup \{x \mid F(x)>0\}$ and $\delta=$ $\inf \{x \mid F(x)<1\}$. We further assume that $E(X)$ exists.

Lemma 4.1. Under the Assumption 4.1, if $E(X \mid X \leq x)=g(x) \tau(x)$, where $\tau(x)=\frac{f(x)}{F(x)}$ and $g(x)$ is a continuous differentiable function of $x$ with the condition that $\int_{\gamma}^{x} \frac{u-g^{\prime}(u)}{g(u)} d u$ is finite for all $x, \gamma<x<\delta$, then $f(x)=$ $c e^{\int_{\gamma}^{x}} \frac{u-g^{\prime}(u)}{g(u)} d u$, where $c$ is determined by the condition $\int_{\gamma}^{\delta} f(x) d x=1$.

Proof. follows from Ahsanullah and Shakil [7]. 
Lemma 4.2. Under the Assumption 4.1, if $E(X \mid X \geq x)=g(x) r(x)$, where $r(x)=\frac{f(x)}{1-F(x)}$ and $g(x)$ is a continuous differentiable function of $x$ with the condition that $\int_{x}^{\delta} \frac{u+g^{\prime}(u)}{g(u)} d u$ is finite for all $x, \gamma<x<\delta$, then $f(x)=c e^{-\int_{x}^{\delta} \frac{u+g^{\prime}(u)}{g(u)} d u}$, where $c$ is determined by the condition $\int_{\gamma}^{\delta} f(x) d x=1$.

Proof. follows from Ahsanullah and Shakil [7].

4.1. Characterization by Truncated Moment. The following two theorems are based on truncated moment.

Theorem 4.1. Suppose that $X$ is an absolutely continuous random variable with $c d f F(x)$ with $F(\mu-\sigma)=0, F(\mu+\sigma)=1$, and $E(X)$ exists. Then $E(X \mid X \leq x)=g(x) \tau(x)$, where

$$
g(x)=\frac{\left\{\frac{\alpha\left(x^{2}-\sigma^{2}\right)}{2}-\frac{\alpha \mu^{2}}{2}+\alpha \mu \sigma\right\}+\frac{\lambda \sigma x}{\pi} \sin \left(\frac{(x-\mu) \pi}{\sigma}\right)+\frac{\lambda \sigma^{2}}{\pi^{2}}\left[1+\cos \left(\frac{(x-\mu) \pi}{\sigma}\right)\right]}{\left[\alpha+\lambda \cos \left(\frac{(x-\mu) \pi}{\sigma}\right)\right]},
$$

and $\tau(x)=\frac{f(x)}{F(x)}$, if and only if $f(x)=\frac{1}{2 \alpha \sigma}\left(\alpha+\lambda \cos \left(\frac{(x-\mu) \pi}{\sigma}\right)\right)$.

Proof. Since $E(X \mid X \leq x)=\frac{\int_{\gamma}^{x} \theta f(\theta) d \theta}{F(x)}$ and $\tau(x)=\frac{f(x)}{F(x)}$, we have

$$
g(x)=\frac{\int_{\gamma}^{x} \theta f(\theta) d \theta}{f(x)} .
$$

Now, if the random variable $X$ satisfies the Assumption 4.1 and has the generalized raised cosine distribution, then

$$
\begin{aligned}
g(x) & =\frac{\int_{\mu-\sigma}^{x} \theta f(\theta) d \theta}{f(x)}=\frac{\int_{\mu-\sigma}^{x} \theta\left(\alpha+\lambda \cos \left(\frac{(\theta-\mu) \pi}{\sigma}\right)\right) d \theta}{\left(\alpha+\lambda \cos \left(\frac{(\theta-\mu) \pi}{\sigma}\right)\right)} \\
& =\frac{\left\{\frac{\alpha\left(x^{2}-\sigma^{2}\right)}{2}-\frac{\alpha \mu^{2}}{2}+\alpha \mu \sigma\right\}+\frac{\lambda \sigma x}{\pi} \sin \left(\frac{(x-\mu) \pi}{\sigma}\right)+\frac{\lambda \sigma^{2}}{\pi^{2}}\left[1+\cos \left(\frac{(x-\mu) \pi}{\sigma}\right)\right]}{\left[\alpha+\lambda \cos \left(\frac{(x-\mu) \pi}{\sigma}\right)\right]}
\end{aligned}
$$

Thus, the proof of (if) part of the Theorem 4.1 follows from Lemma 4.1. Conversely, we will now prove the (only if) condition of Theorem 4.1. Suppose that

$$
g(x)=\frac{\left\{\frac{\alpha\left(x^{2}-\sigma^{2}\right)}{2}-\frac{\alpha \mu^{2}}{2}+\alpha \mu \sigma\right\}+\frac{\lambda \sigma x}{\pi} \sin \left(\frac{(x-\mu) \pi}{\sigma}\right)+\frac{\lambda \sigma^{2}}{\pi^{2}}\left[1+\cos \left(\frac{(x-\mu) \pi}{\sigma}\right)\right]}{\left[\alpha+\lambda \cos \left(\frac{(x-\mu) \pi}{\sigma}\right)\right]} .
$$

Differentiating both sides of the above equation with respect to $x$, we have

or

$$
g^{\prime}(x)=x+g(x) \frac{\frac{\lambda \pi}{\sigma} \sin \left(\frac{(x-\mu) \pi}{\sigma}\right)}{\alpha+\lambda \cos \left(\frac{(x-\mu) \pi}{\sigma}\right)},
$$

$$
\frac{x-g^{\prime}(x)}{g(x)}=-\frac{\frac{\lambda \pi}{\sigma} \sin \left(\frac{(x-\mu) \pi}{\sigma}\right)}{\alpha+\lambda \cos \left(\frac{(x-\mu) \pi}{\sigma}\right)} .
$$


Then, by Lemma 4.1, we obtain

$$
\frac{f^{\prime}(x)}{f(x)}=\frac{x-g^{\prime}(x)}{g(x)}=-\frac{\frac{\lambda \pi}{\sigma} \sin \left(\frac{(x-\mu) \pi}{\sigma}\right)}{\alpha+\lambda \cos \left(\frac{(x-\mu) \pi}{\sigma}\right)} .
$$

On integrating both sides of the above equation with respect to $x$, we obtain

$$
f(x)=c\left[\alpha+\lambda \cos \left(\frac{(x-\mu) \pi}{\sigma}\right)\right]
$$

where $c$ is a constant to be determined. Using the boundary conditions $F(\mu-\sigma)=0$ and $F(\mu+\sigma)=1$, we have $c=\frac{1}{2 \alpha \sigma}$. This completes the proof of Theorem 4.1 .

Theorem 4.2. If the random variable $X$ satisfies the Assumption 4.1 with $\gamma=\mu-\sigma$ and $\delta=\mu+\sigma$, then $E(X \mid X \geq x)=$ $h(x) r(x)$, where $r(x)=\frac{f(x)}{1-F(x)}$ and

$$
h(x)=\frac{(\mu-g(x) f(x))(2 \alpha \sigma)}{\left[\alpha+\lambda \cos \left(\frac{(x-\mu) \pi}{\sigma}\right)\right]}
$$

where $g(x)$ is given by Eq. (4.1) and

$$
E(X)=\mu \text {, if and only if } f(x)=\frac{1}{2 \alpha \sigma}\left(\alpha+\lambda \cos \left(\frac{(x-\mu) \pi}{\sigma}\right)\right) .
$$

Proof. Suppose that $E(X \mid X \geq x)=h(x) r(x)$. Then, since $E(X \mid X \geq x)=\frac{\int_{x}^{\delta} \theta f(\theta) d \theta}{1-F(x)}$ and $r(x)=\frac{f(x)}{1-F(x)}$, we have $h(x)=\frac{\int_{x}^{\delta} \theta f(\theta) d \theta}{f(x)}$. Now, if the random variable $X$ satisfies the Assumption 4.1 and has the generalized raised cosine distribution, then we have

$$
\begin{aligned}
h(x) & =\frac{\int_{x}^{\mu+\sigma} \theta f(\theta) d \theta \quad \int_{\mu-\sigma}^{\mu+\sigma} \theta f(\theta) d \theta-\int_{\mu-\sigma}^{x} \theta f(\theta) d \theta}{f(x)}=\frac{(E)}{\left[\alpha+\lambda \cos \left(\frac{(x-\mu) \pi}{\sigma}\right)\right]} \\
& =\frac{(\mu-g(x) f(x))(2 \alpha \sigma)}{\left[\alpha+\lambda \cos \left(\frac{(x-\mu) \pi}{\sigma}\right)\right]}
\end{aligned}
$$

This completes the proof of (if) part. Conversely, we will now prove the (only if) condition of Theorem 4.2 . Suppose that

$$
h(x)=\frac{(\mu-g(x) f(x))(2 \alpha \sigma)}{\left[\alpha+\lambda \cos \left(\frac{(x-\mu) \pi}{\sigma}\right)\right]} .
$$

Differentiating both sides of the above equation with respect to $x$ and simplifying, we have

$$
h^{\prime}(x)=-x+h(x) \frac{\frac{\lambda \pi}{\sigma} \sin \left(\frac{(x-\mu) \pi}{\sigma}\right)}{\alpha+\lambda \cos \left(\frac{(x-\mu) \pi}{\sigma}\right)},
$$


or

$$
\frac{x-h^{\prime}(x)}{h(x)}=\frac{\frac{\lambda \pi}{\sigma} \sin \left(\frac{(x-\mu) \pi}{\sigma}\right)}{\alpha+\lambda \cos \left(\frac{(x-\mu) \pi}{\sigma}\right)}
$$

Then, by Lemma 4.2, we obtain

$$
\frac{f^{\prime}(x)}{f(x)}=-\frac{x+h^{\prime}(x)}{h(x)}=-\frac{\frac{\lambda \pi}{\sigma} \sin \left(\frac{(x-\mu) \pi}{\sigma}\right)}{\alpha+\lambda \cos \left(\frac{(x-\mu) \pi}{\sigma}\right)} .
$$

which, on integrating with respect to $x$, gives

$$
f(x)=c\left[\alpha+\lambda \cos \left(\frac{(x-\mu) \pi}{\sigma}\right)\right]
$$

where $c$ is a constant to be determined. Using the boundary conditions $F(\mu-\sigma)=0$ and $F(\mu+\sigma)=1$, we have $c=\frac{1}{2 \alpha \sigma}$. This completes the proof of Theorem 4.2 .

4.2. Characterizations by Order Statistics. Here, we will provide the characterizations based on order statistics, for which we first recall the following well-known results. Let $X_{1}, X_{2}, \ldots, X_{n}$ be $n$ independent copies of the random variable $X$ having absolutely continuous distribution function $F(x)$ and pdf $f(x)$. Suppose that $X_{1, n} \leq$ $X_{2, n} \leq \ldots \leq X_{n, n}$ are the corresponding order statistics. It is known that $X_{j, n} \mid X_{k, n}$, for $1 \leq k<j \leq n$, is distributed as the $(j-k)$ th order statistics from $(n-k)$ independent observations from the random variable $V$ having the pdf $f_{V}(v \mid x)$ where $f_{V}(v \mid x)=\frac{f(v)}{1-F(x)}, 0 \leq v<x$, see, for example, Ahsanullah et al. [3], chapter 5, or Arnold et al. [8], chapter 2, among others. Further, $X_{i, n} \mid X_{k, n}=x$, for $1 \leq i<k \leq n$ is distributed as ith order statistics from $k$ independent observations from the random variable $W$ having the pdf $f_{W}(w \mid x)$ where $f_{W}(w \mid x)=\frac{f(w)}{F(x)}, w<x$. Let $S_{k-1}=\frac{1}{k-1}\left(X_{1, n}+X_{2, n}+\ldots+X_{k-1, n}\right)$ and $T_{k, n}=\frac{1}{n-1}\left(X_{k+1, n}+X_{k+2, n}+\ldots+X_{n, n}\right)$.

In the following two theorems, we will provide the characterizations of the generalized raised cosine distribution, $X \sim \operatorname{GENRCD}(\alpha, \lambda, \mu, \sigma)$, based on order statistics.

Theorem 4.3. Suppose the random variable $X$ satisfies the Assumption 4.1 with $\gamma=\mu-\sigma$ and $\delta=\mu+\sigma$, then $E\left(S_{k-1} \mid X_{k, n}=x\right)=g(x) \tau(x)$, where $\tau(x)=\frac{f(x)}{F(x)}$ and

$$
g(x)=\frac{\left\{\frac{\alpha\left(x^{2}-\sigma^{2}\right)}{2}-\frac{\alpha \mu^{2}}{2}+\alpha \mu \sigma\right\}+\frac{\lambda \sigma x}{\pi} \sin \left(\frac{(x-\mu) \pi}{\sigma}\right)+\frac{\lambda \sigma^{2}}{\pi^{2}}\left[1+\cos \left(\frac{(x-\mu) \pi}{\sigma}\right)\right]}{\left[\alpha+\lambda \cos \left(\frac{(x-\mu) \pi}{\sigma}\right)\right]},
$$

if and only if $f(x)=\frac{1}{2 \alpha \sigma}\left(\alpha+\lambda \cos \left(\frac{(x-\mu) \pi}{\sigma}\right)\right)$.

Proof. It is known from Ahsanullah et al. [3], and David and Nagaraja [9] that $E\left(S_{k-1} \mid X_{k, n}=x\right)=E(X \mid X \leq x)$. The result follows from Theorem 4.1.

Theorem 4.4. Suppose the random variable $X$ satisfies the Assumption 4.1 with $\gamma=\mu-\sigma$ and $\delta=\mu+\sigma$, then $E\left(T_{k, n} \mid X_{k, n}=x\right)=h(x) r(x)$, where $r(x)=\frac{f(x)}{1-F(x)}$ and $h(x)=\frac{(\mu-g(x) f(x))(2 \alpha \sigma)}{\left[\alpha+\lambda \cos \left(\frac{(x-\mu) \pi}{\sigma}\right)\right]}$, if and only if $f(x)=\frac{1}{2 \alpha \sigma}\left(\alpha+\lambda \cos \left(\frac{(x-\mu) \pi}{\sigma}\right)\right)$.

Proof. It is known from Ahsanullah et al. [3], and David and Nagaraja [9] that $E\left(T_{k, n} \mid X_{k, n}=x\right)=E(X \mid X \geq x)$. The result follows from Theorem 4.2. 
4.3. Characterization by Upper Record Values. Here, we will provide the characterizations based on upper record values, for which we first recall the following definitions. Suppose that $X_{1}, X_{2}, \ldots$ is a sequence of independent and identically distributed absolutely continuous random variables with distribution function $F(x)$ and pdf $f(x)$. Let $Y_{n}=\max \left(X_{1}, X_{2}, \ldots, X_{n}\right)$ for $n \geq 1$. We say that $X_{j}$ is an upper record value of $\left\{X_{n}, n \geq 1\right\}$, if, $Y_{j}>Y_{j-1}$, $j>1$. The indices at which the upper records occur are given by the record times

$$
\left\{U(n)>\min \left(j \mid j>U(n+1), X_{j}>X_{U(n-1)}, n>1\right\} \text { and } U(1)=1 .\right.
$$

We will denote the $n$th upper record value as $X(n)=X_{u(n)}$. For details, see for example Ahsanullah [1], among others. In the following theorem, we will provide the characterization of the generalized raised cosine distribution, $X \sim \operatorname{GENRCD}(\alpha, \lambda, \mu, \sigma)$, based on upper record values.

Theorem 4.5. Suppose the random variable $X$ satisfies the Assumption 4.1 with $\gamma=\mu-\sigma$ and $\delta=\mu+\sigma$, then $E(X(n+$ 1) $\mid X(n)=x)=h(x) r(x)$, where $r(x)=\frac{f(x)}{1-F(x)}$, where $h(x)=\frac{(\mu-g(x) f(x))(2 \alpha \sigma)}{\left[\alpha+\lambda \cos \left(\frac{(x-\mu) \pi}{\sigma}\right)\right]}$, if and only if $f(x)=\frac{1}{2 \alpha \sigma}\left(\alpha+\lambda \cos \left(\frac{(x-\mu) \pi}{\sigma}\right)\right)$.

Proof. It is known from Ahsanullah et al. [3], and Nevzorov [15] that

$$
E(X(n+1) \mid X(n)=x)=E(X \mid X \geq x) .
$$

The result follows from Theorem 4.2.

\section{Percentile Points}

The percentile points of the generalized raised cosine distribution, $X \sim \operatorname{GENRCD}(\alpha, \lambda, \mu, \sigma)$, are computed in this section, with the pdf (2.1) and cdf (2.2). For any $0<p<1$, the $100 p$ th percentile (also called the quantile of order $p$ ) of the generalized raised cosine distribution, $X \sim \operatorname{GENRCD}(\alpha, \lambda, \mu, \sigma)$, is a number $x_{p}$ such that the area under $f_{X}(x)$ to the left of $x_{p}$ is $p$. That is, $x_{p}$ is any root of the equation given by

$$
F\left(x_{p}\right)=\int_{\mu-\sigma}^{x_{p}} f_{X}(u) d u=p .
$$

The percentile points $x_{p}$ associated with the cdf of $X \sim \operatorname{GENRCD}(\alpha, \lambda, \mu, \sigma)$, that is

$$
F(x)=\frac{1}{2}\left[1+\frac{x-\mu}{\sigma}+\frac{\lambda}{\alpha \pi} \sin \left(\frac{(x-\mu) \pi}{\sigma}\right)\right],
$$

are computed numerically, which are provided in the Table 1 below. 


\begin{tabular}{|c|c|c|c|c|c|c|c|c|}
\hline Percentiles $p$ & & 0.7 & 0.75 & 0.8 & 0.85 & 0.9 & 0.95 & 0.99 \\
\hline \multicolumn{9}{|l|}{ Parameters } \\
\hline $\begin{array}{l}\alpha=1, \lambda=1 \\
\mu=0.5, \sigma=0.5\end{array}$ & $x_{p}$ & 0.10358 & 0.13237 & 0.16368 & 0.19896 & 0.24109 & 0.29804 & 0.38400 \\
\hline $\begin{array}{l}\alpha=1, \lambda=1 \\
\mu=0, \sigma=1\end{array}$ & $x_{p}$ & 0.20716 & 0.26474 & 0.32737 & 0.39792 & 0.48219 & 0.59608 & 0.76801 \\
\hline $\begin{array}{l}\alpha=1, \lambda=1 \\
\mu=0, \sigma=1.5\end{array}$ & $x_{p}$ & 0.31074 & 0.39711 & 0.49105 & 0.59688 & 0.72328 & 0.89412 & 1.15200 \\
\hline $\begin{array}{l}\alpha=1, \lambda=1 \\
\mu=0, \sigma=2\end{array}$ & $x_{p}$ & 0.41432 & 0.52948 & 0.65473 & 0.79584 & 0.96438 & 1.1922 & 1.53360 \\
\hline $\begin{array}{l}\alpha=1, \lambda=1 \\
\mu=0, \sigma=2.5\end{array}$ & $x_{p}$ & 0.51790 & 0.66185 & 0.81842 & 0.99180 & 1.20550 & 1.49020 & 1.92000 \\
\hline $\begin{array}{l}\alpha=1, \lambda=1 \\
\mu=0, \sigma=3\end{array}$ & $x_{p}$ & 0.62148 & 0.79423 & 0.98210 & 1.19380 & 1.44660 & 1.78820 & 2.3040 \\
\hline $\begin{array}{l}\alpha=1, \lambda=1 \\
\mu=0, \sigma=3.5\end{array}$ & $x_{p}$ & 0.72505 & 0.92660 & 1.14580 & 1.39270 & 1.68770 & 2.08630 & 2.68800 \\
\hline $\begin{array}{l}\alpha=1, \lambda=1 \\
\mu=0, \sigma=4\end{array}$ & $x_{p}$ & 0.82863 & 1.05901 & 1.20950 & 1.59170 & 1.92880 & 2.38430 & 3.07200 \\
\hline $\begin{array}{l}\alpha=1, \lambda=1 \\
\mu=0, \sigma=4.5\end{array}$ & $x_{p}$ & 0.93221 & 1.19130 & 1.47320 & 1.79060 & 2.16980 & 2.68240 & 3.45600 \\
\hline $\begin{array}{l}\alpha=1, \lambda=1 \\
\mu=0, \sigma=5\end{array}$ & $x_{p}$ & 1.03580 & 1.32370 & 1.63680 & 1.98960 & 2.441090 & 2.9804 & 3.84000 \\
\hline
\end{tabular}

TABLe 1. Percentile Points of the generalized raised cosine distribution, $X \sim$ $\operatorname{GENRCD}(\alpha, \lambda, \mu, \sigma)$

\section{Estimation of Parameters}

6.1. The Method of Moments. When $n$ is a positive integer, using the pdf (2.1) of $X \sim \operatorname{GENRCD}(\alpha, \lambda, \mu, \sigma)$, we have the following expression for the $n$th Moments:

$$
\begin{aligned}
E\left(X^{n}\right)= & \int_{\mu-\sigma}^{\mu+\sigma} x^{n}\left[\frac{1}{2 \alpha \sigma}\left(\alpha+\lambda \cos \left(\frac{(x-\mu) \pi}{\sigma}\right)\right)\right] d x \\
= & \frac{1}{2 \sigma(n+1)}\left[(\mu+\sigma)^{n+1}-(\mu-\sigma)^{n+1}\right] \\
& +\left(\frac{\lambda}{2 \alpha \sigma}\right) \int_{\mu-\sigma}^{\mu+\sigma} x^{n} \cos \left(\frac{(x-\mu) \pi}{\sigma}\right) d x . \\
= & \frac{1}{2 \sigma(n+1)}\left[(\mu+\sigma)^{n+1}-(\mu-\sigma)^{n+1}\right] \\
& +\left(\frac{\lambda}{2 \alpha \pi}\right) \int_{-\pi}^{\pi}\left(\mu+\frac{\sigma}{\pi} u\right)^{n} \cos u d u,
\end{aligned}
$$


taking $\frac{(x-\mu) \pi}{\sigma}=u$ in the integral in (6.1). Now, in the last expression for $E\left(X^{n}\right)$, taking $n=1,2,3,4$, and evaluating the respective integrals numerically, we obtain the first four moments respectively as follows:

$$
\begin{aligned}
& E(X)=\frac{4 \mu \sigma}{4 \sigma}+\frac{\lambda}{2 \alpha \pi}\left(\int_{-\pi}^{\pi} \mu \cos u d u+\frac{\sigma}{\pi} \int_{-\pi}^{\pi} u \cos u d u\right)=\mu ; \\
& E\left(X^{2}\right)= \frac{1}{6 \sigma}\left(6 \mu^{2} \sigma+2 \sigma^{3}\right) \\
&+\frac{\lambda}{2 \alpha \pi}\left(\int_{-\pi}^{\pi} \mu^{2} \cos u d u+\int_{-\pi}^{\pi} \frac{2 \sigma \mu}{\pi} u \cos u d u+\int_{-\pi}^{\pi} \frac{\sigma^{2}}{\pi^{2}} u^{2} \cos u d u\right) \\
&= \mu^{2}+\frac{\sigma^{2}}{3} \frac{\lambda}{2 \alpha \pi} \frac{\sigma^{2}}{\pi^{2}}(-4 \pi)=\mu^{2}+\frac{1}{3} \sigma^{2}-\frac{2 \lambda \sigma^{2}}{\alpha \pi^{2}} ; \\
& E\left(X^{3}\right)= \frac{1}{8 \sigma}\left(8 \mu^{3} \sigma+8 \mu \sigma^{3}\right) \\
&+\frac{\lambda}{2 \alpha \pi}\left(\int_{-\pi}^{\pi} \mu^{3} \cos u d u+\int_{-\pi}^{\pi} \frac{3 \sigma \mu}{\pi} u \cos u d u+\frac{3 \sigma^{2} \mu}{\pi^{2}} \int_{-\pi}^{\pi} u^{2} \cos u d u\right. \\
&\left.\quad+\frac{\sigma^{2}}{\pi^{3}} \int_{-\pi}^{\pi} u^{3} \cos u d u\right) \\
&= \mu\left(\sigma^{2}+\mu^{2}\right)+\frac{\lambda}{2 \alpha \pi} \frac{3 \sigma^{2} \mu}{\pi^{2}}(-4 \pi)=\mu^{3}-\frac{6 \lambda \mu \sigma^{2}}{\alpha \pi^{2}} ;
\end{aligned}
$$

and

$$
\begin{aligned}
E\left(X^{4}\right)= & \frac{1}{10 \sigma}\left(10 \mu^{4} \sigma+20 \mu^{2} \sigma^{3}+2 \sigma^{5}\right) \\
& +\frac{\lambda}{2 \alpha \pi}\left(\int_{-\pi}^{\pi} \mu^{4} \cos u d u+\frac{4 \mu^{3} \sigma}{\pi} \int_{-\pi}^{\pi} u \cos u d u+\frac{6 \mu^{2} \sigma^{2}}{\pi^{2}} \int_{-\pi}^{\pi} u^{2} \cos u d u\right. \\
& \left.\quad+\frac{4 \mu \sigma^{3}}{\pi^{3}} \int_{-\pi}^{\pi} u^{3} \cos u d u+\frac{\sigma^{4}}{\pi^{4}} \int_{-\pi}^{\pi} u^{4} \cos u d u\right) \\
= & \frac{1}{5} \sigma^{4}+2 \sigma^{2} \mu^{2}+\mu^{4}+\frac{\lambda}{2 \alpha \pi}\left(\frac{6 \mu^{2} \sigma^{2}}{\pi^{2}}(-4 \pi)+\frac{\sigma^{4}}{\pi^{4}}\left(48 \pi-8 \pi^{3}\right)\right) \\
= & \mu^{4}+2 \sigma^{2} \mu^{2}\left(1-\frac{6 \lambda}{\alpha \pi^{2}}\right)+\sigma^{4}\left(\frac{24 \lambda}{\alpha \pi^{4}}-\frac{4 \lambda}{\alpha \pi^{2}}+\frac{1}{5}\right) .
\end{aligned}
$$

Thus, the moment estimation (MMEs) of the parameters $\alpha, \lambda, \mu$ and $\sigma$ can be determined by solving above the system of four equations by Newton-Raphson's iteration method of solving system of non-linear equations using the computer packages such as Maple, or R, MathCAD, or other software.

6.2. The Method of Maximum Likelihood. Given a sample $x_{i}, i=1,2,3, \ldots, n$, the likelihood function of (2.1) is given by $L=\prod_{i=1}^{n} f\left(x_{i}\right)$. The objective of the likelihood function approach is to determine those values of the parameters that maximize the function $L$. Suppose $R=\ln (L)=\sum_{i=1}^{n} \ln \left[f\left(x_{i}\right)\right]$. Then the maximum likelihood estimates (MLE) of the parameters $\alpha, \lambda, \mu$ and $\sigma$ are obtained by solving the maximum likelihood equations

$$
\frac{\partial R}{\partial \alpha}=0, \frac{\partial R}{\partial \lambda}=0, \frac{\partial R}{\partial \mu}=0 \text { and } \frac{\partial R}{\partial \sigma}=0,
$$

using the Newton-Raphson's iteration method of solving system of nonlinear equations with the help of computer packages such as Maple, or R, MathCAD14, or other software.

\section{Applications}

Here, we show some applications of our proposed distribution. 
7.1. Example 1. In this example, we use a real data set to illustrate the potentiality and the performance of the generalized raised cosine distribution, $X \sim \operatorname{GENRCD}(\alpha, \lambda, \mu, \sigma)$, by considering the following Average Monthly Temperature in Denver, Colorado, USA, in Table 2, reported by Sullivan [17, p. 590]. As pointed out by Sullivan [17], "Since the data represent average monthly temperatures collected over many years, the data will not vary much from year to year and so will essentially repeat each year. In other words, the data are periodic". The scatter plot of these data is provided in Figure 4.

\begin{tabular}{|l|c|}
\hline Month, $\mathbf{x}$ & Average Monthly Temperature, $^{\circ} \mathbf{F}$ \\
\hline January, 1 & 30.7 \\
\hline February, 2 & 32.5 \\
\hline March, 3 & 40.4 \\
\hline April, 4 & 47.4 \\
\hline May, 5 & 57.1 \\
\hline June, 6 & 67.4 \\
\hline July, 7 & 74.2 \\
\hline August, 8 & 72.5 \\
\hline September, 9 & 62.4 \\
\hline October, 10 & 50.9 \\
\hline November, 11 & 38.3 \\
\hline December, 12 & 30.0 \\
\hline
\end{tabular}

TABLE 2. Average Monthly Temperature in Denver, Colorado, USA (Source: US National Oceanic and Atmospheric Administration) 


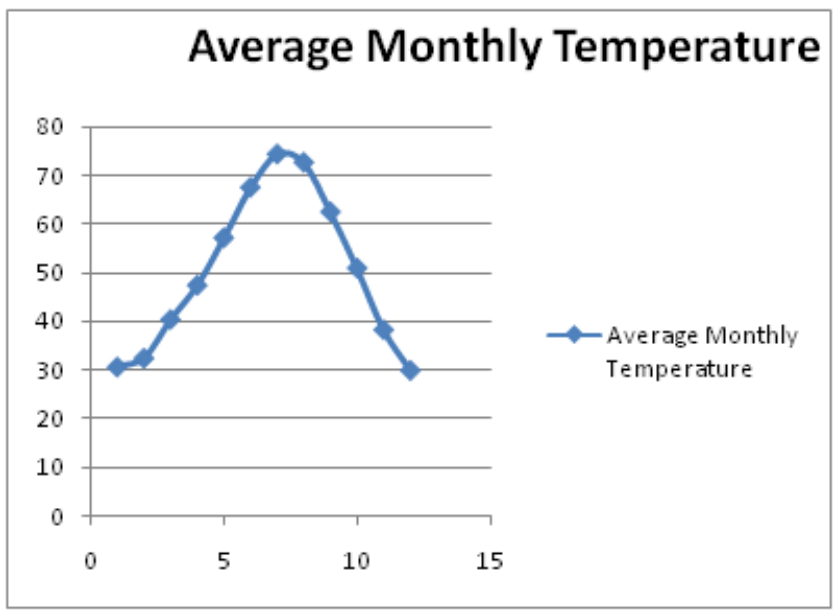

FiguRE 4. Scatter Plot of Average Monthly Temperature

The mean, median, standard deviation, skewness and kurtosis of this data are $50.32,49.15,16.27,0.18$, and -1.50 respectively. Following the technique of fitting the sinusoidal functions to the periodic data, the estimate of the unknown parameters of the generalized raised cosine distribution,

$$
X \sim \operatorname{GENRCD}(\alpha, \lambda, \mu, \sigma),
$$

are calculated as follows: $(\alpha=52.1, \lambda=22.1, \mu=7, \sigma=6)$. Thus, for these computed values of the estimated parameters, the pdf and cdf of the generalized raised cosine distribution, $X \sim \operatorname{GENRCD}(\alpha, \lambda, \mu, \sigma)$, are respectively given by:

and

$$
f(x)=\frac{1}{625.2}\left[52.1+(22.1) \cos \left(\frac{(x-7) \pi}{6}\right)\right]
$$

It is easy to see that

$$
F(x)=\frac{1}{2}\left[1+\frac{x-7}{6}+\frac{0.424}{\pi} \sin \left(\frac{(x-7) \pi}{6}\right)\right] .
$$

$$
\int_{1}^{13} \frac{1}{625.2}\left[52.1+(22.1) \cos \left(\frac{(x-7) \pi}{6}\right)\right] d x=1
$$

The graphs for the scatter diagram of the data corresponding to the above expression for the pdf is shown in the Figure 5 below. 


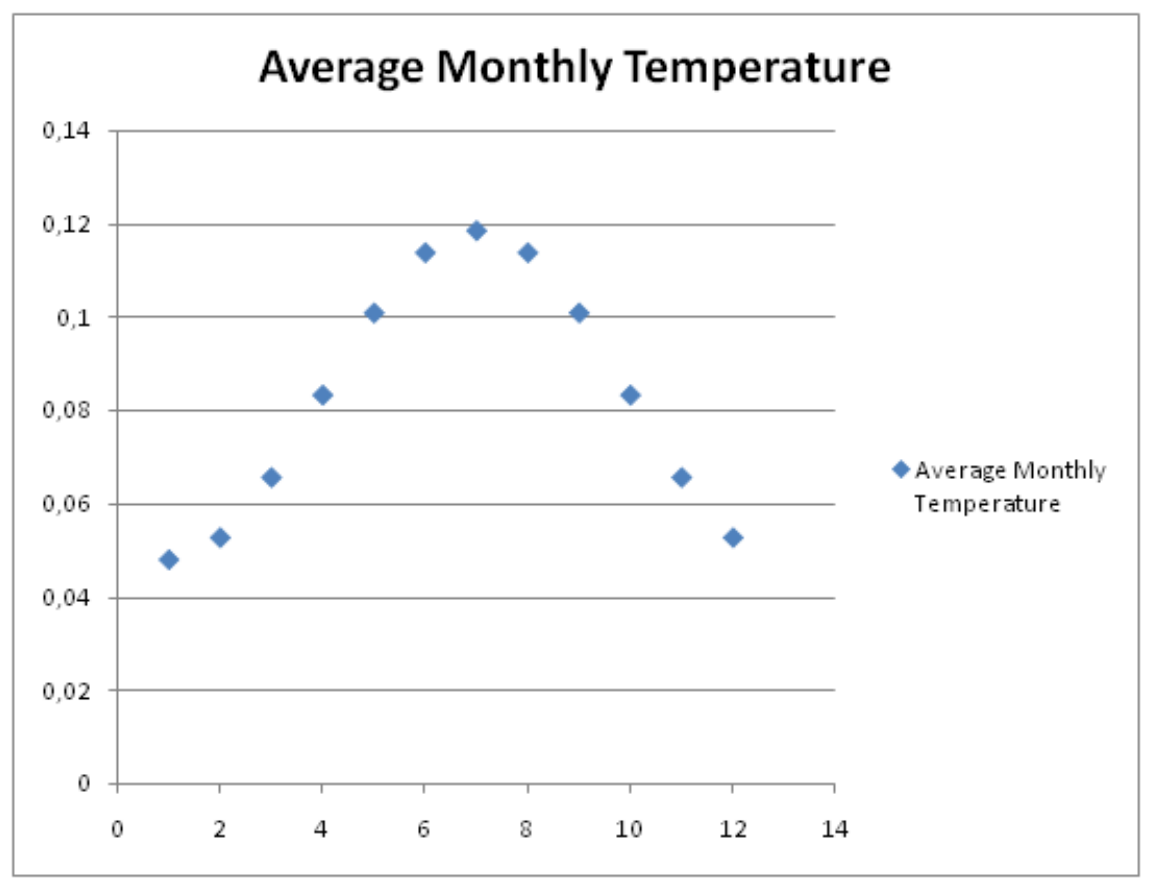

FiguRE 5. Scatter Diagram of Data in Table 2 Corresponding to the pdf $f(x)=$ $\frac{1}{625.2}\left[52.1+(22.1) \cos \left(\frac{(x-7) \pi}{6}\right)\right]$

The graphs for the above pdf and cdf are shown in the Figure 6 and Figure 7 respectively. 
ON A GENERALIZED RAISED COSINE DISTRIBUTION

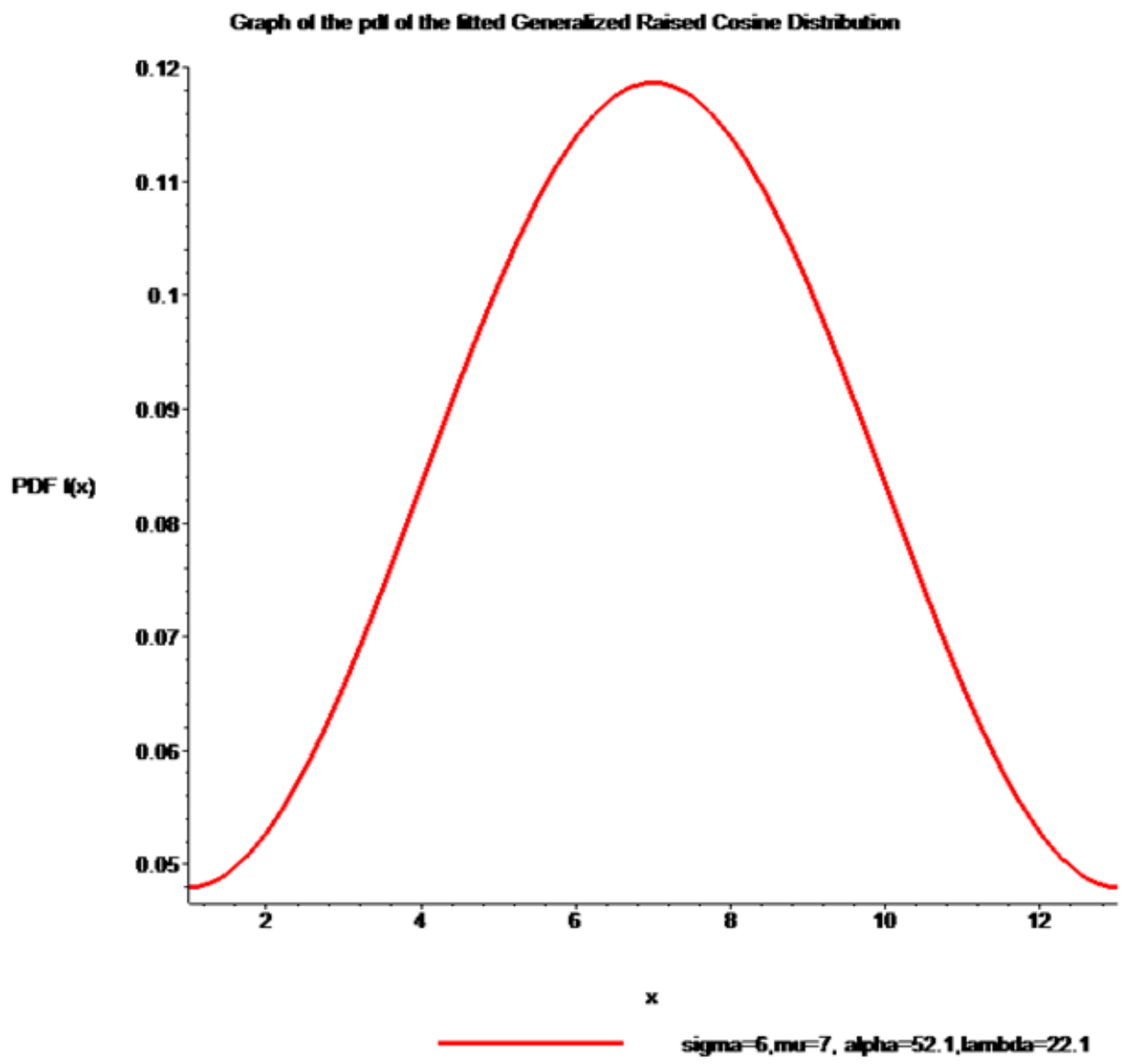

Figure 6. Plots of the pdf, $f(x)=\frac{1}{625.2}\left[52.1+(22.1) \cos \left(\frac{(x-7) \pi}{6}\right)\right]$. 
Graph of the call of the Gted Generalized Raised Cosine Distribution

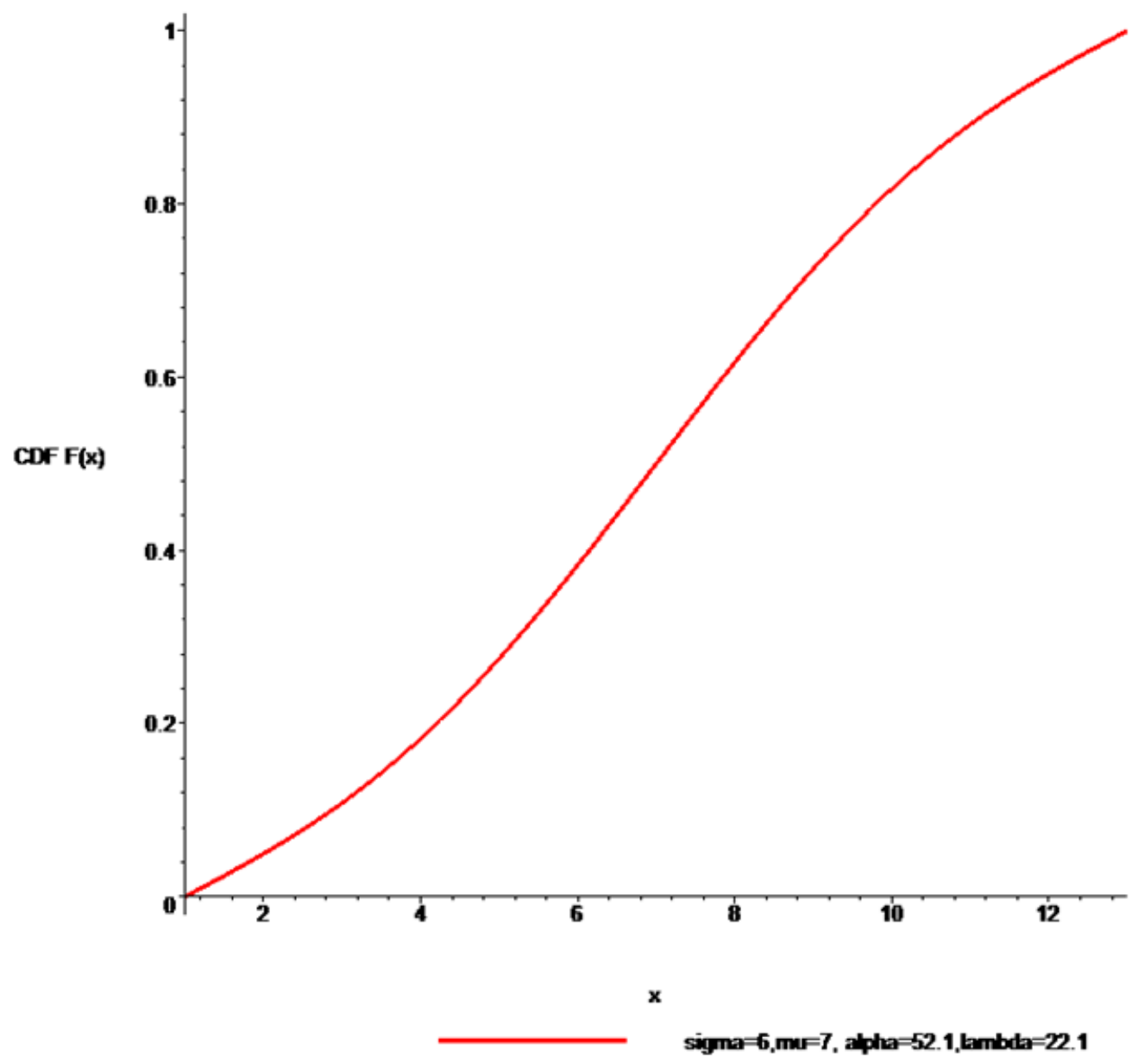

FIGURE 7. Plots of the cdf, $F(x)=\frac{1}{2}\left[1+\frac{x-7}{6}+\frac{0.424}{\pi} \sin \left(\frac{(x-7) \pi}{6}\right)\right]$.

It is observed from Figure 6 that the pdf, $f(x)=\frac{1}{625.2}\left[52.1+(22.1) \cos \left(\frac{(x-7) \pi}{6}\right)\right]$, for our new model, $X \sim$ $\operatorname{GENRCD}(\alpha=52.1, \lambda=22.1, \mu=7, \sigma=6)$, fits reasonably well to the empirical scatter diagram in Figure 5 .

7.2. Example 2. In this example, we use a real data set to illustrate the potentiality and the performance of the generalized raised cosine distribution, $X \sim \operatorname{GENRCD}(\alpha, \lambda, \mu, \sigma)$, by considering the Average Monthly Daylight Hours in Nashville, Tennessee, USA, (Source: https://www.timeanddate.com/sun/usa/nashville and http://aa.usno.navy.mil/data/docs/RS-OneYear.php), in the Table 3. As pointed out above, these data are also periodic in nature. The scatter plot diagram of these data is provided in Figure 8. 


\begin{tabular}{|l|c|}
\hline Month, $\mathbf{x}$ & Average Monthly Daylight Hours \\
\hline January, 1 & 9.98 \\
\hline February, 2 & 10.89 \\
\hline March, 3 & 11.97 \\
\hline April, 4 & 13.13 \\
\hline May, 5 & 14.1 \\
\hline June, 6 & 14.62 \\
\hline July, 7 & 14.37 \\
\hline August, 8 & 13.52 \\
\hline September, 9 & 12.4 \\
\hline October, 10 & 11.26 \\
\hline November, 11 & 10.25 \\
\hline December, 12 & 9.72 \\
\hline
\end{tabular}

TABLE 3. Average Monthly Daylight Hours in Nashville, Tennessee, USA

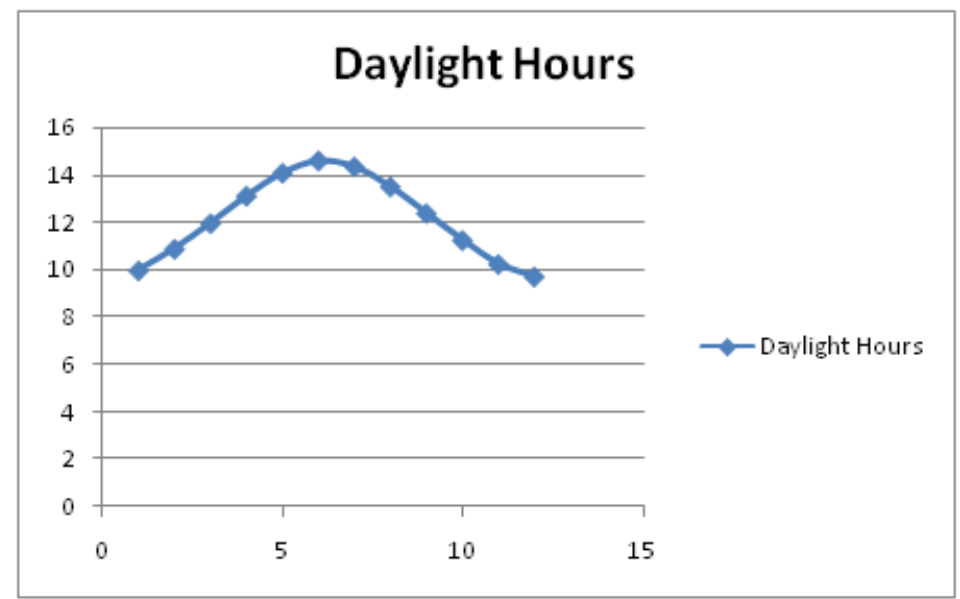

FiguRE 8. Scatter Plot of Average Monthly Daylight Hours

The mean, median, standard deviation, skewness and kurtosis of this data are 12.184, 12.185, 1.77, -0.014 , and -1.55 respectively. Following the technique of fitting the sinusoidal functions to the periodic data, the estimate of the unknown parameters of the generalized raised cosine distribution, $X \sim \operatorname{GENRCD}(\alpha, \lambda, \mu, \sigma)$, are calculated as follows:

$(\alpha=12.17, \lambda=2.45, \mu=7, \sigma=6)$. Thus, for these computed values of the estimated parameters, the pdf and cdf of the generalized raised cosine distribution, $X \sim \operatorname{GENRCD}(\alpha, \lambda, \mu, \sigma)$, are respectively given by:

and

$$
f(x)=\frac{1}{146.04}\left[12.17+(2.45) \cos \left(\frac{(x-7) \pi}{6}\right)\right]
$$

$$
F(x)=\frac{1}{2}\left[1+\frac{x-7}{6}+\frac{0.201}{\pi} \sin \left(\frac{(x-7) \pi}{6}\right)\right] .
$$


It is easy to see that

$$
\int_{1}^{13} \frac{1}{146.04}\left[12.17+(2.45) \cos \left(\frac{(x-7) \pi}{6}\right)\right] d x=1
$$

The graphs for the scatter diagram of the data corresponding to the above expression for the pdf is shown in the Figure 9 .

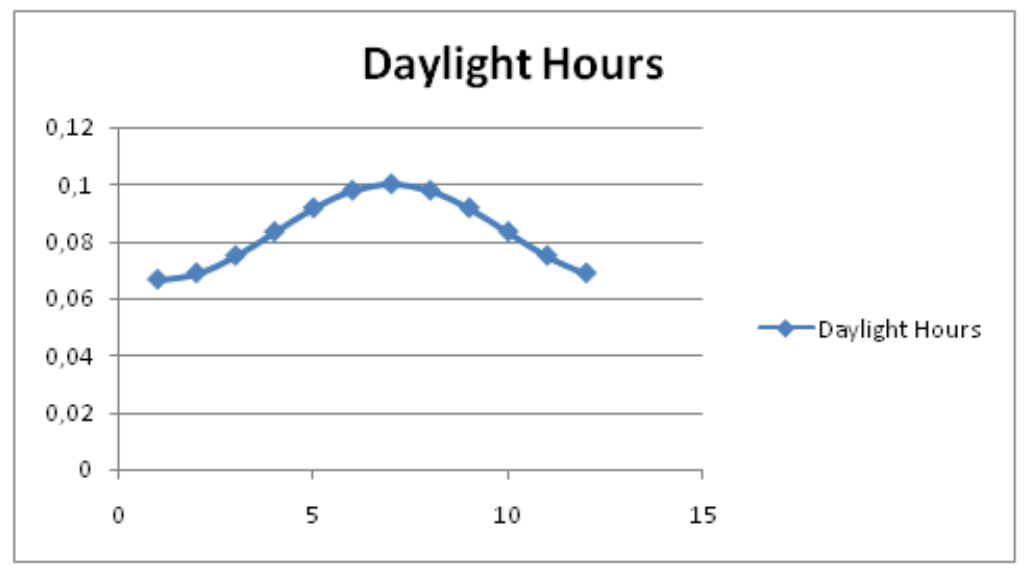

Figure 9. Scatter Diagram of Data in Table 3 Corresponding to the pdf $f(x)=$ $\frac{1}{146.04}\left[12.17+(2.45) \cos \left(\frac{(x-7) \pi}{6}\right)\right]$

The graphs for the above pdf and cdf are shown in the Figure 10 and the Figure 11 respectively. 
ON A GENERALIZED RAISED COSINE DISTRIBUTION

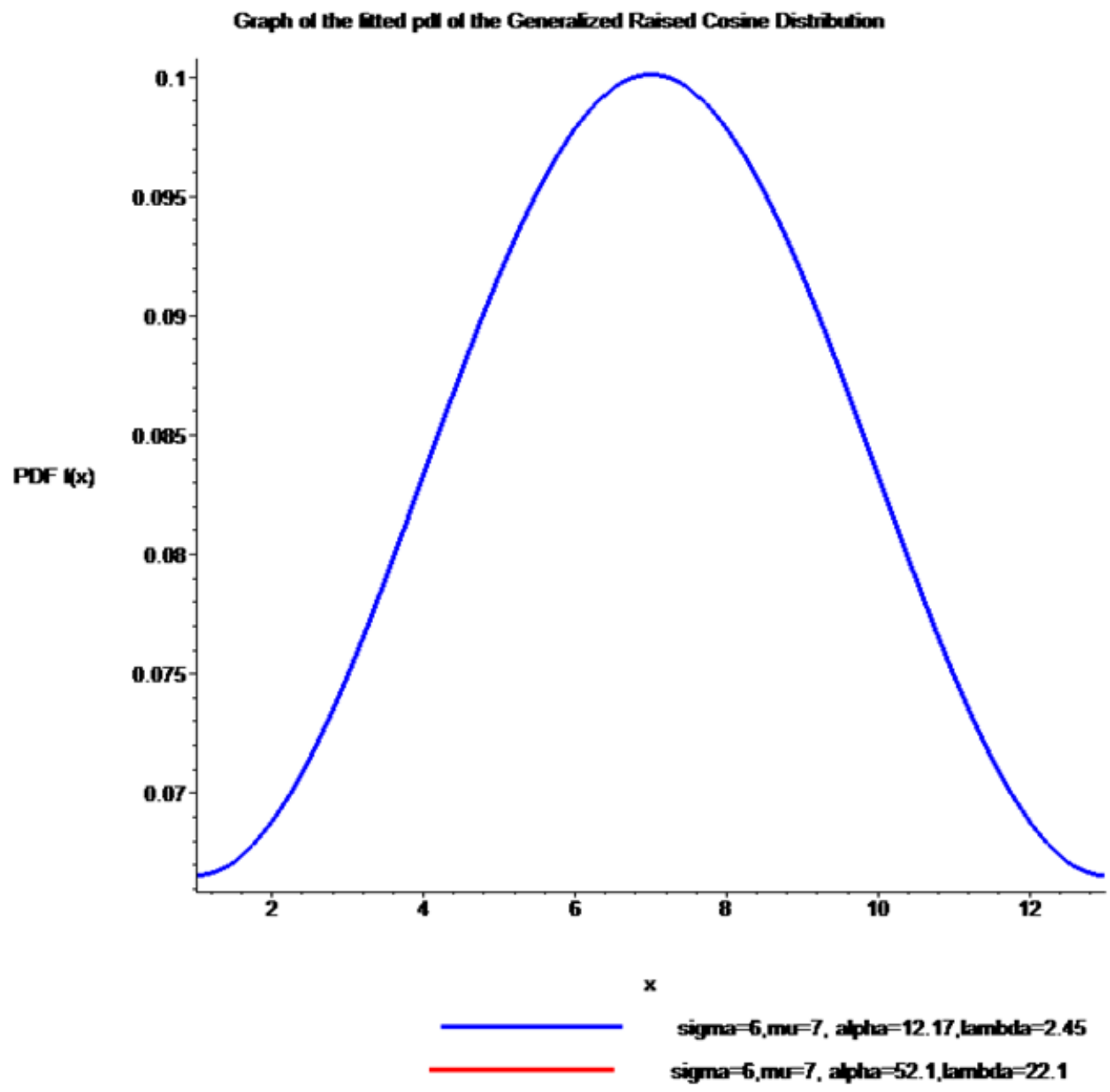

FIGURE 10. Plots of the pdf, $f(x)=\frac{1}{146.04}\left[12.17+(2.45) \cos \left(\frac{(x-7) \pi}{6}\right)\right]$. 


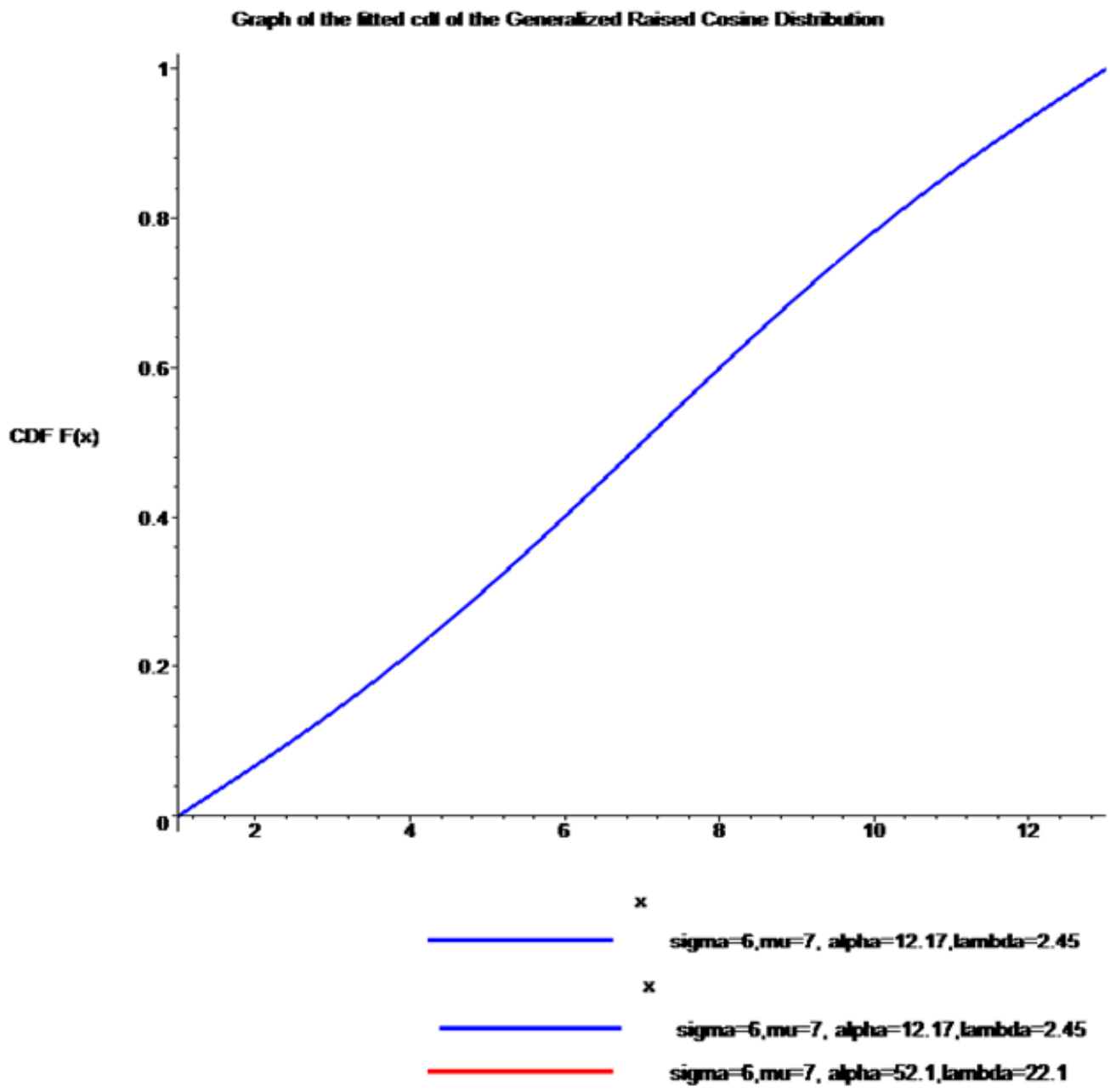

FigUre 11. Plots of the cdf, $F(x)=\frac{1}{2}\left[1+\frac{x-7}{6}+\frac{0.201}{\pi} \sin \left(\frac{(x-7) \pi}{6}\right)\right]$.

It is observed from Figure 10 that the pdf,

$$
f(x)=\frac{1}{146.04}\left[12.17+(2.45) \cos \left(\frac{(x-7) \pi}{6}\right)\right]
$$

for our new model, $X \sim \operatorname{GENRCD}(\alpha=12.17, \lambda=2.45, \mu=7, \sigma=6)$, fits reasonably well to the empirical scatter diagram in Figure 9.

\section{Concluding Remarks}

This paper introduces a generalized raised cosine distribution. Several distributional properties of the proposed distribution have been given. It is observed that our proposed distribution endures most of the properties of symmetric distributions. Also, some new characterizations of the generalized raised cosine distribution by truncated moment, order statistics and upper record values are obtained. We have computed percentiles and shown some applications. We sincerely believe that the paper will be quite useful for researchers and practitioners in the fields of probability, statistics, and other applied sciences. 


\section{References}

1. Ahsanullah, M., Record Statistics, Nova Science Publishers, New York, USA. (1995).

2. Ahsanullah, M., Characterizations of Univariate Continuous Distributions, Atlantis-Press, Paris, France. (2017).

3. Ahsanullah, M., Nevzorov,V. B., and Shakil, M., An Introduction to Order Statistics, Atlantis-Press, Paris, France. (2013).

4. Ahsanullah, M., Kibria, B. M. G., and Shakil, M., Normal and Student's $t$ Distributions and Their Applications, Atlantis Press, Paris, France. (2014).

5. Ahsanullah, M., Shakil, M., and Kibria, B. G.,Characterizations of folded student's t distribution. Journal of Statistical Distributions and Applications, 2(1), 15. (2015).

6. Ahsanullah, M., Shakil, M., Kibria, B. M., Characterizations of Continuous Distributions by Truncated Moment. Journal of Modern Applied Statistical Methods, 15(1), 17. (2016).

7. Ahsanullah, M., and Shakil, M., Some Characterizations of Raised Cosine Distribution. Journal of Advanced Statistics and Probability, 6(2), 42-49. (2018).

8. Arnold, B.C., Balakrishnan, and Nagaraja, H. N., First Course in Order Statistics, Wiley, New York, USA.(2005 ).

9. David, H. A., and Nagaraja, H. N., Order Statistics, Third Edition, Wiley, New York, USA. (2003).

10. Galambos, J., and Kotz, S., Characterizations of probability distributions. A unified approach with an emphasis on exponential and related models, Lecture Notes in Mathematics, 675, Springer, Berlin. (1978).

11. King, M., Statistics for Process Control Engineers: A Practical Approach, First Edition, Wiley, New York, USA. (2017).

12. Kotz, S., and Shanbhag, D. N., Some new approaches to probability distributions. Advances in Applied Probability, 12, 903 - 921. (1980).

13. Kyurkchiev, V., and Kyurkchiev, N., On the approximation of the step function by raised-cosine and laplace cumulative distribution functions. European International Journal of Science and Technology, 4(9), 75 - 84. (2016).

14. Nagaraja, H., Characterizations of Probability Distributions. In Springer Handbook of Engineering Statistics (pp. 79 95), Springer, London, UK. (2006).

15. Nevzorov, V. B., Records: Mathematical Theory, Translation of Mathematical Monograph. American Mathematical Society, Rhode Island, USA. (2001).

16. Rinne, H., LocationVScale Distribution: Linear Estimation and Probability Plotting Using MATLAB, Copyright: Prof. em. Dr Hors Rinne, Department of Economics and Management Science, JustusVLiebligVUniversity, Giessen, Germany.(2010).

17. Sullivan, M., Algebra and Trigonometry, Pearson Education, Inc., Tenth Edition, New York, USA.(2016).

18. Willink, R., Measurement Uncertainty and Probability, Cambridge University Press, First Edition, New York, USA.(2013). 